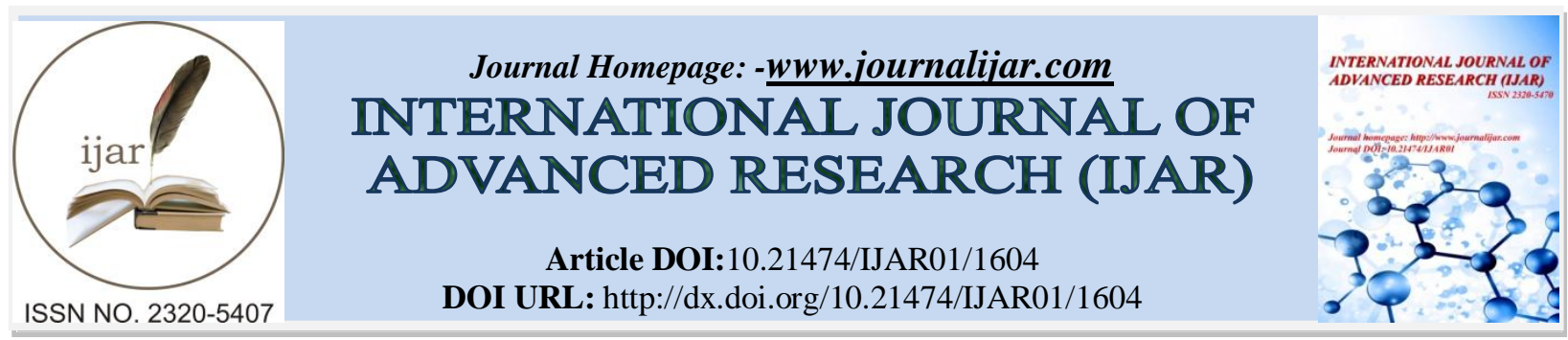

RESEARCH ARTICLE

\title{
BREAST DENSE CELL COUNT AND ANALYSIS IN DIGITAL MAMMOGRAM BY AUTO THRESHOLD METHOD.
}

Dr. (Mrs). D. Pugazhenthi ${ }^{1}$ and Mrs. N. M. Sangeetha ${ }^{2}$.

1. Assitant .Professor PG \& Research Dept. of Computer Science, Quaid-E-Millath Govt.College for Women (A), Chennai, India.IEEE MEMBER.

2. Research Scholar, PG \& Research Dept. of Computer Science, Quaid-E-Millath Govt. College for Women (A), Chennai, India.IEEE MEMBER.

\section{Manuscript Info}

(..........................

Manuscript History

Received: 12 July 2016

Final Accepted: 23 August 2016

Published: September 2016

Key words:-

Digital Mammogram, Filters, Breast

Abnormalities.

\section{Abstract}

Breast cancer one of the most leading disease among the women's. In this research paper, we count the number of abnormal breast cells and find their position with image processingtechniques. The proposed work contains four stages, and there are i) smoothing ii) Threshold iii) Basic Morphology iv) Partial analysis. The particular cells segregation is the most important process of this work. In present work is the application of various filters used in image processing and apply these filters in detecting the highly dense cells responsible for breast abnormalities. Two different type of filteroperators in image processing are Gradient, and Laplacian operators have been used and implemented. The LABVIEW and MATLABSoftware gave practical usages to this image processing system because it can interconnect with other tools used in this system and controls them to have an automatic system. This experimental result is the potential effectiveness of such a system on diagnostic tasks that require the classification of individual cells. The digital mammogram images and data sample have been taken from the online database has been made for the detection of dense cells responsible for breast abnormalities. The main aim of this paper helps the radiologist to detect the breast dense cells.

Copy Right, IJAR, 2016,. All rights reserved.

\section{Introduction:-}

To evaluate the results of this method, an approach for the detection of breast abnormalities of digital mammogram was also proposed. This research article presents an automated procedure for breast cell counting and its performance. The procedure for analyzing dense breast cancer cell image consists of four procedures, i.e. i) smoothing ii) Threshold iii) Basic Morphology iv) Partial analysis. The goal of detection and segmentation is to locate and extract a highly dense cell from the image. In digital mammogram images, this detection and segmentation play important roles in breast cancer classification between dense and non-dense [1]. Computing technology has often proven useful in performing tedious or complex tasks fast, accurately. In extensive previous work by this author and others, computational intelligence was used to identify breast lesions based on radiologist's impresses of various features visible on a digital mammogram images.[2] In other areas of medicine, computational aptitude has been used to segment an image into its constituent parts, permitting automated assessments of tissue

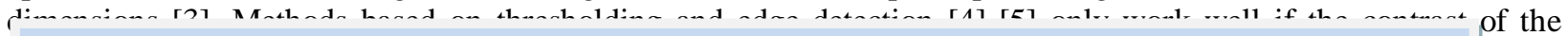

Corresponding Author:-Dr.(Mrs).D.Pugazhenthi.

Address:-Assitant .Professor PG \& Research Dept.of Computer Science, Quaid-E-Millath Govt. 
image is brilliant. However in most cases, this technique yields broken edges and poor detection in noisy locations. Watershed techniques [6]-[7] have also been functional to the problem of cell separation. If the rises in the image are large enough, then the background can be easily separated from the background image [8].

The methodology that is used in this research paper is based on image processing tools such as image improvements (filter, noise removal, particle analysis, etc.), image segmentation, vision assistant and LABVIEW software [9]. The most important property of this method is accuracy where preserves its difficulty. Also, noise removal algorithm that is used in this system for improving the counting ability of the breast cells is one of the most significant qualities of this research work.

\section{Cancer Cell Image Analysis:-}

In the image preprocessing, the Filters: Smoothing - Gaussian transform is performed. The global thresholding and morphological operations are performed in segmentation. The segmentation results show that the average performance is $85 \%$ when square-shaped structuring element. The experimental results show that the average performance is more than $90 \%$.

\section{DATASET DESCRIPTION:-}

The digital mammogram is the low energy $x$-ray of the breast. It is one of the efficient screening tools which compare to another screening tool. The digital mammogram images taken by an online database.

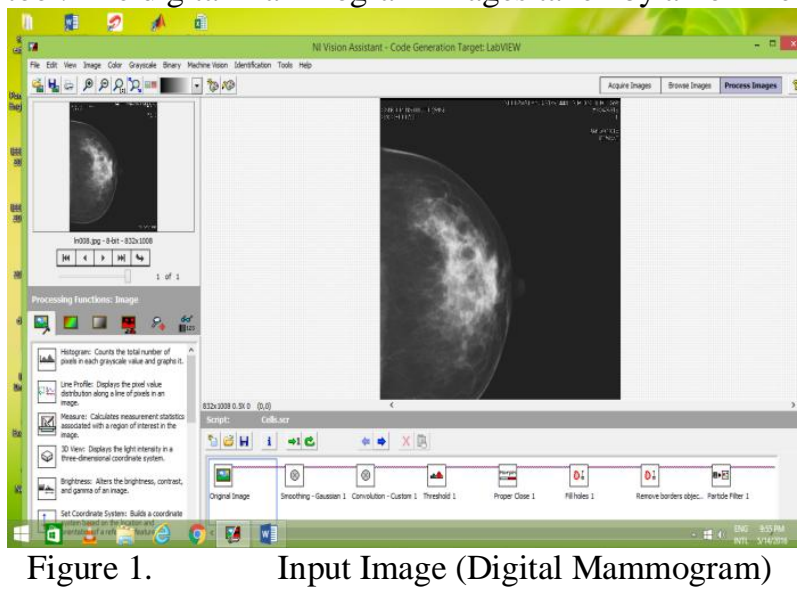

\section{Proposed Methodology:-}

All the digital mammogram image have to be transferred into LABVIEW Software. After that, the image processing process will be continued by using LABVIEW and MATLAB together. The image enhancement, segmentation, etc. are done with this two software. The system of this process is shown in Fig. 1. In Fig. 2 the original digital mammogram image that is used for image processing system is shown. In this picture, particular cells are shown beside the normal cells. Fig. 3 shows it will look that some of the cells are marked. These are the defected cells, and the goal is to mark them between other cells in the digital mammogram image with image processing. 


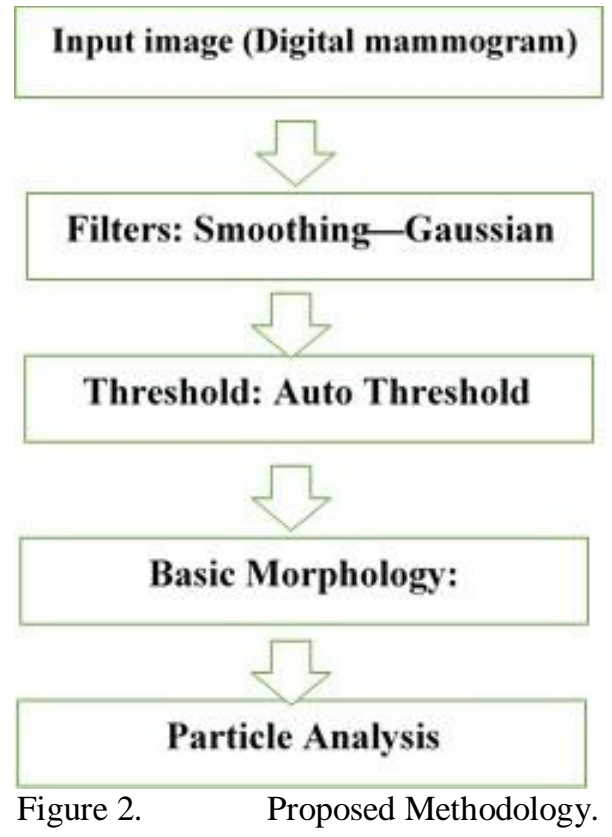

The cell analysis example counts the number of cells and measures their areas using the Grayscale Filtering, Thresholding, and Binary Image Inversion functions.

\section{Filters:-}

Smoothing - Gaussian - Smoothes the image using a Gaussian filter. The effect of Gaussian smoothing is to blur a picture, in a similar approach to the mean filter. The degree of smoothing is determined by the standard deviation of the Gaussian. The Gaussian smoothing process yields a "weighted average" of each pixel's region, with the average weighted more towards the value of the central pixels. This is in contrast to the mean filter's uniformly weighted average. Because of this, a Gaussian provides moderate smoothing and reserves edge better than a similarly sized low screen. As can be seen, the dense cells are extracted and highlighted among the other cells, very well. This technique can be used in large scale cells check and can improve the accuracy and speed of the examination and counting process of the highly dense affected cells.

By counting the detected cells changes of the effect of medical treatment on the growth of the cancer cells.

\section{Filters: Edge Detection-Laplacian:-}

Highlights the edges in the image using a Laplacian filter. This operation finds edges in the filtered image and adds them to the original image. This process helps increase the separation between the cells and the background.

The filters are used in the course of identifying the image by locating the sharp edges which are discontinuous. These breaks bring changes in pixels strengths which define the boundaries of the object. The object is breast, and a new methodology is applied to identify the breast type using its morphological features. Here, it is applied to different two-dimensional filters, comparative studies and displays the result. In this edge detection method, the assumption edges are the pixels with a high gradient. A fast rate of change of intensity at some direction is given by the angle of the gradient vector is observed at edge pixels. In Fig. 3, an ideal edge pixel and the corresponding gradient vector are shown. At the pixel, the intensity changes from 0 to 255 at the direction of the slope. The magnitude of the angle indicates the strength of the edge. Calculation of the slope at uniform regions and end up with a 0 vector which means there is no edge pixel. In natural images which usually do not have the ideal discontinuity or the uniform regions as in the Fig. 1 and the process the magnitude of the gradient to make a decision to detect the edge pixels. The fundamental processing is applied for a threshold. If the gradient scale is larger than the threshold, decide the method in matches on the brink pixel. An edge pixel is described by using two important features, primarily the edge strength, which is equal to the magnitude of the gradient and secondarily edge direction, which is equal to the angle of the slope. A slope is not defined at all for a discrete function. Instead, the slope, which 
can be set for the ideal continuous image is estimated using some operators. Among these operators "Roberts, Sobel and Prewitt" are gradient-based edge detector.

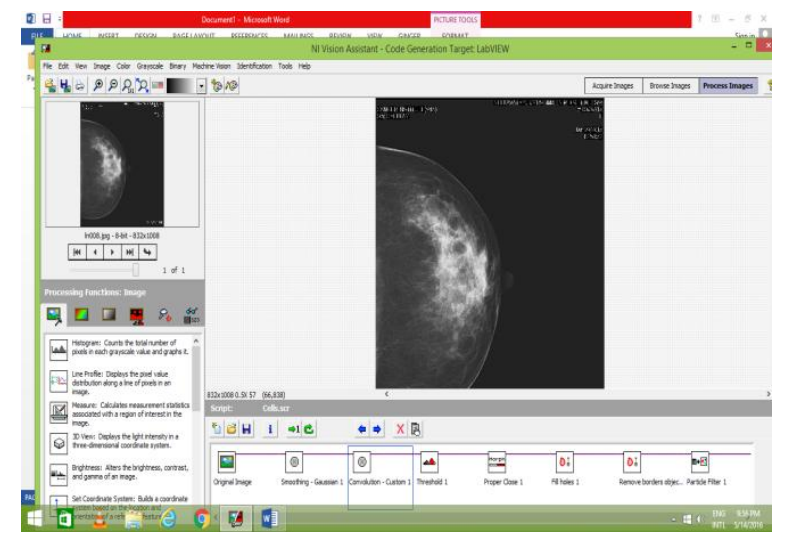

Threshold:-

Figure 3. Representing Edge Detection Using Laplacian Filter.

Auto Threshold-Metric-Separates the dark regions in the digital image from the rest of the image using an automatic threshold technique. In this case, the dark regions in the digital mammogram image correspond to the cells. The regions corresponding to the medium in which the cells are present appear as foreground in the resulting binary image

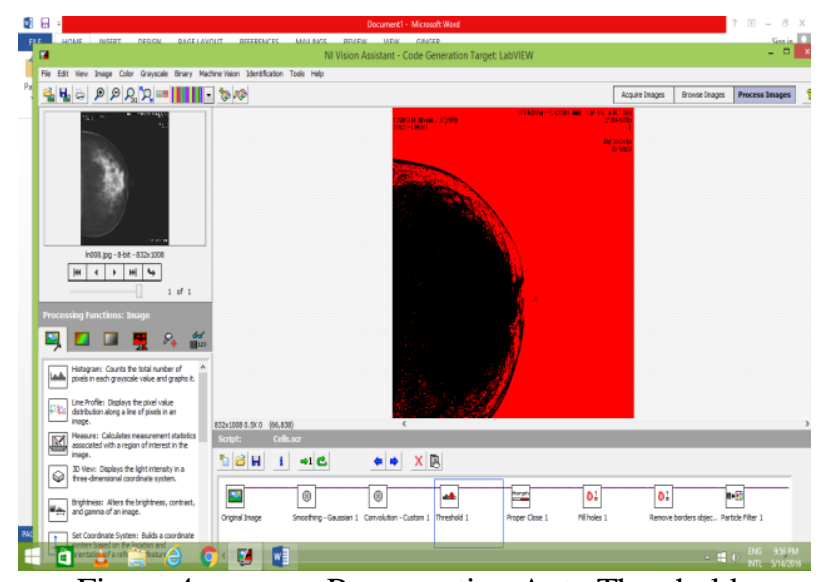

Figure $4 . \quad$ Representing Auto Threshold.

\section{Basic Morphology:-}

Proper Close-improves the shape of the particles in the binary image by smoothing the boundary of the particles, filling small holes in the particles, and closing small gaps along the perimeter of the particles. Laplacian Edge Detection It wishes to build a morphing process which operates on Features extracted from target images automatically. It can be a good beginning to find the edges of the target digital mammogram images 
Adv. Morphology:-

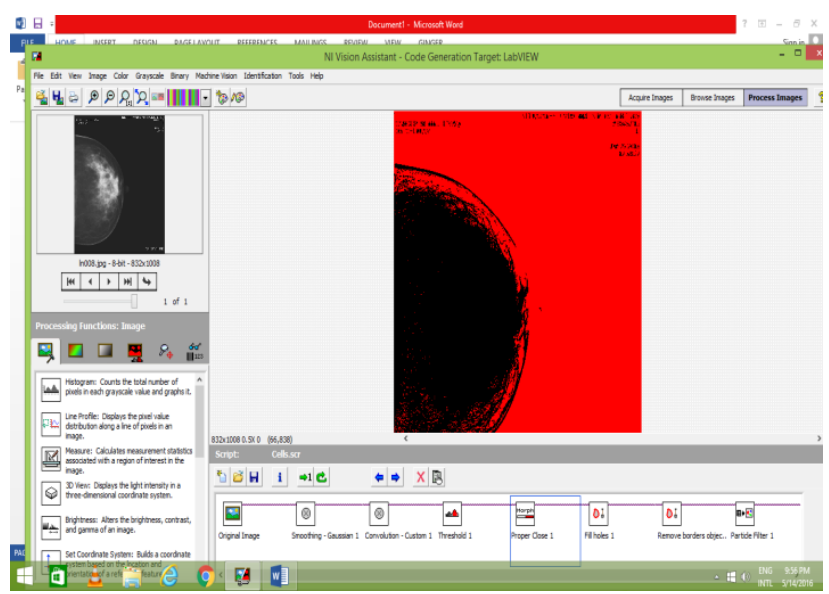

Figure $5 . \quad$ Representing proper close morphology operation.

Fill Holes-Advance morphology is the one of the essential parts to fills any size holes in a particle. This process helps to differentiate between the sizes of the breast cells.

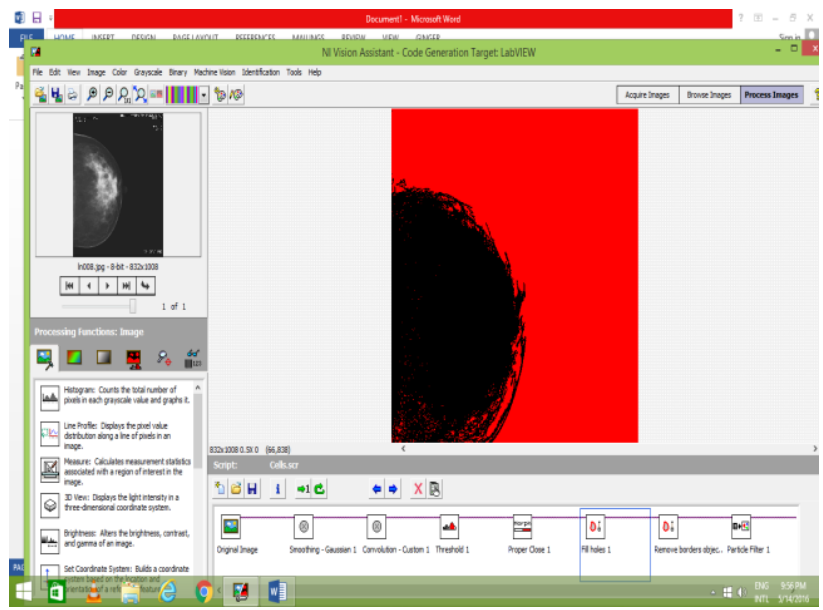

Figure 6. $\quad$ Representing Advance Morphology Using Fill Hole Operation.

\section{Particle Filter:}

Removes unwanted particles from the binary image using the particle filter. This application only analyzes particles that are mostly circular and larger than 10 pixels. Particles that have a Heywood Circularity Factor that falls outside of the 0 to 1.40 range or have a pixel area of less than ten are removed. 


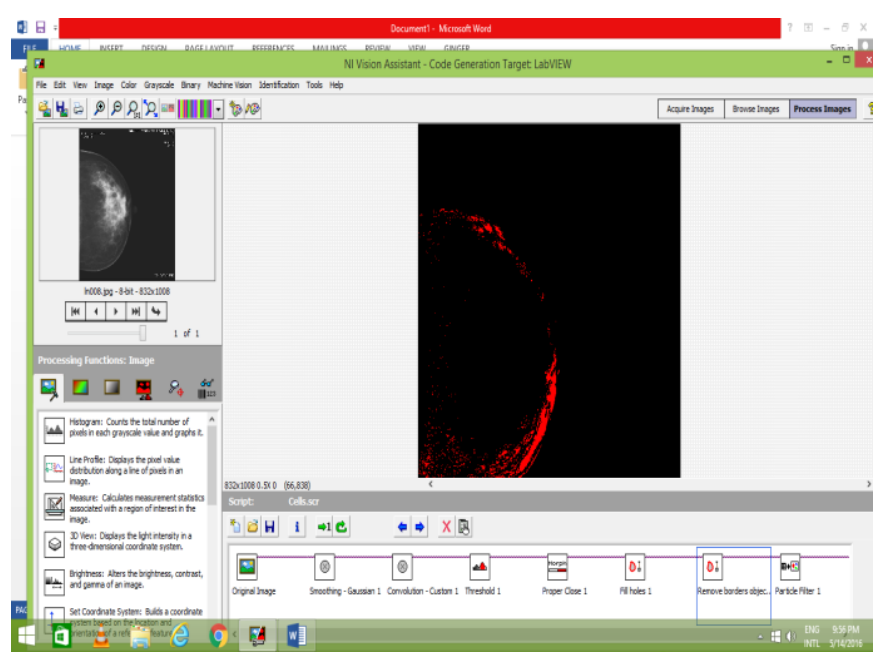

Figure $7 . \quad$ Representing particle filters

Particle Analysis:-

Analyzes the properties of the remaining particles (cells) in the image. The particle measurement function can analyze up to 50 different properties of a particle.

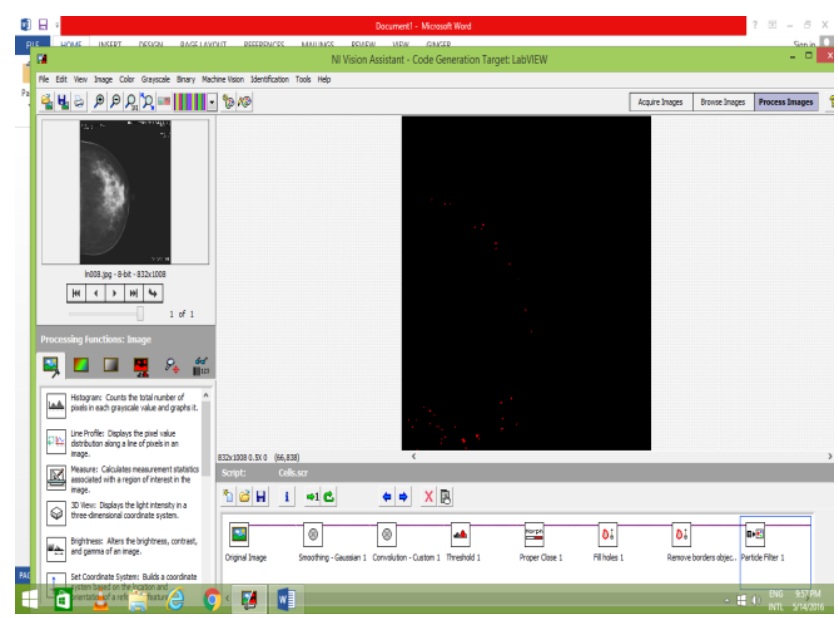

Figure $8 . \quad$ Representing the Dense Cells.

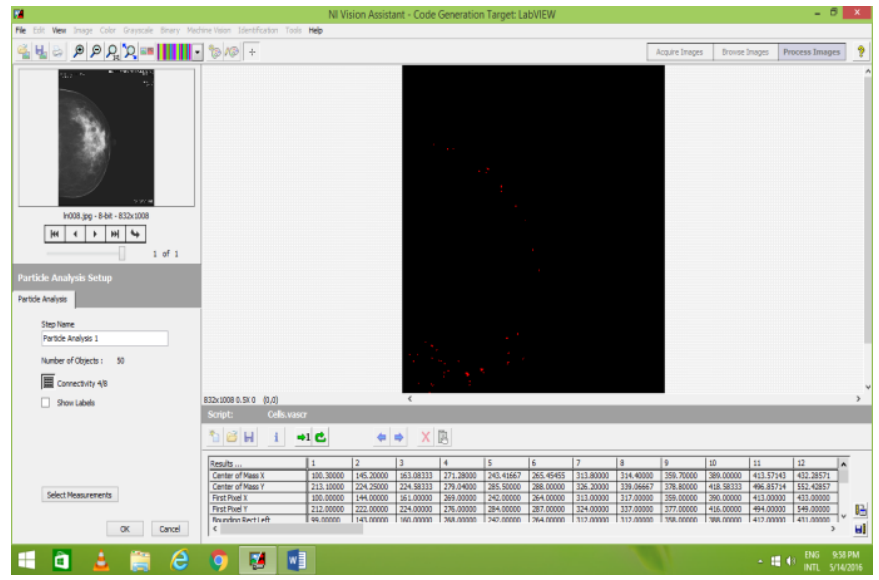

Figure 9. Representing the cells in different color patterns. 


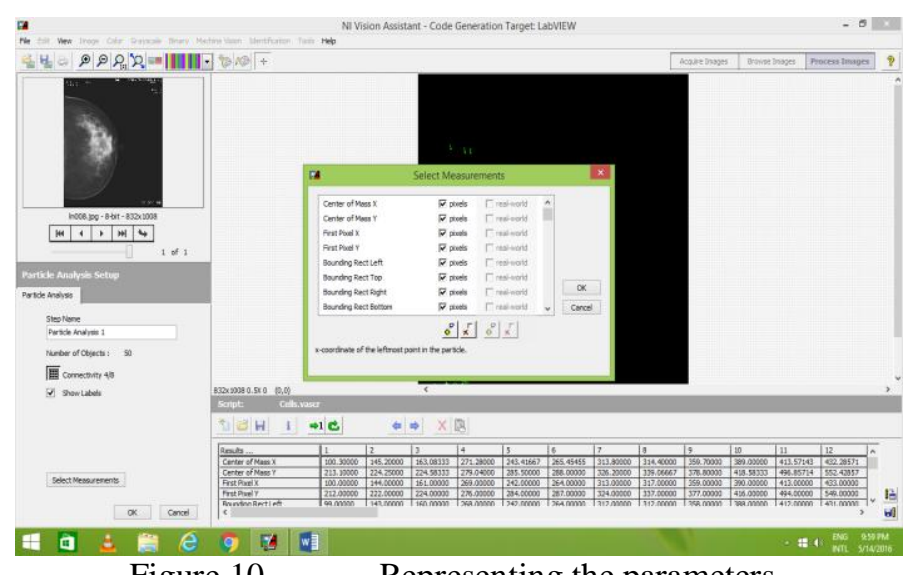

\section{Conclusion:-}

Figure $10 . \quad$ Representing the parameters.

Digital mammogram is the fundamental and efficient screening tool to count and analysis the breast cells. The highly dense cells are extracted and highlighted among the other cells. This process method used in online database evaluation and counting process of the highly dense cells in the breast. By counting and analysis the breast thick cell .The experimental results shows the effect of medical treatment.

\section{References:-}

1. Hossain Ghayoumi Zadeh, Siamak Janianpour -Recognition and classification of the cancer cells by using image processing and LabVIEW, an international journal of computer theory and engineering vol 5, No 1 Feb 2013.

2. P.Phukpattrasonnont, S.Limsirortans and P.Boonyaphiphat Ëvaluation of computer aided analysis for stained breast cancer cell images ", in the 3 nd International Symposium on Bio-medical Engineering,10-11 Nov.2008 pp-213-216.

3. S.prabha, K.Anandth, "Total variation based edge enhance, for level set segmentation and Asymmetry analysis in breast - in engineering in medicine and biology society 2014, 36th international conference of the IEEE 2014."

4. Y.Ramadevi, B.Kalaiyani,"segmentation and object Recognition, using Edge Detection Techniques", International Technology (IJCSIT), Vol 2, No.6 DEC 2010.

5. Lu Wang and Nelson H.C.Yung, Extraction of moving objects from their background based on multiple adaptive threshold and boundary Evaluation ", IEEE Transaction on Multimedia, Vol.14, No.1, March 2010.

6. R.siegel, J.Ma, Z.Zou " cancer statistics 2014"CA:a cancer journal of clinical ,vol-64,No 1,pp9-29,2014

7. Q.Zhou, Z.Li and K.Aggarwal,"Boundary extraction in thermal images by edge map" in Proceeding of 2014, ACM Symposium on Applied Computing ACM.

8. Sangeetha, D.Pugazhenthi,"A Novel Shape And Boundary Extraction Image Processing Technique For Detecting The Breast Abnormalities Using Digital Mammogram -Labview Implementation" In IEEE sponsored 3rd international conference on innovation in information embedded and communication system 2016

9. Sangeetha, D.Pugazhenthi," Breast Tissue Identification in Digital Mammogram Using Edge Detection Techniques. in International Conference on Recent trends in computing technology Breast Tissue Identification in Digital Mammogram Using Edge Detection Techniques. (ICRTCT)feb-2016 